\section{DRAGON BYTES: USING DATA ANALYTICS TO DETERMINE LEARNER INTERESTS}

Stacey Harris, Assim Javaid. UK

10.1136/bmjpo-2021-RCPCH.246

Background A new trainee-oriented paediatric podcast was launched on 8th September 2019; Dragon Bytes. The aim of this was to support trainees more holistically with training. Episodes covering a wide range of topics; complex conditions, reflective writing or career advice for example. The aim of this study is to use Data from streaming websites to determine user demographics and preferences.

Objectives Methods:

User analytics were accessed on Spotify, SoundCloud, apple and google podcasts on 4th January 2021, over one year after launch, for the most current statistics. These were exported to SPSS for analysis.

Results A total of 48 episodes have been released in the first year. With 64 episodes at the time by the data was collected.

Listeners on Spotify are 57\% female and 42\% male. Those aged 28-34 are most likely to listen, accounting for $43 \%$ of listens.

Outside of the UK, the three countries that most listen to the podcasts are the United States, Australia and the Sauda Arabia.

There have been 17,121 listens to the podcasts; 12,386 via SoundCloud and 4,313 via Spotify, 390 on apple podcasts and 38 on google podcasts. The average number of listeners per podcast was 267.5. The most popular podcast was with RCPCH COVID adapted clinical exam with over 1000 listens. 'Paediatric Knowledge' were the most popular group of podcasts with an average of over 7.23 listens/week, the least popular was 'Field Reports' with 2.73 listens/week.

Conclusions A Wales-led educational podcast has an international audience. Having teaching delivered digitally has the advantage of easy access to analytical data that can shape future teaching sessions.

We could use the data to promote the listening further to an international audience and the accessibility of the different podcast platforms.

There is clearly a strong appetite for Dragonbytes podcasts with RCPCH and also theory-based podcasts and so more focus needs to be given to this area in future.

\section{NON-ACCIDENTAL INJURY AND ABUSIVE HEAD TRAUMA IN CHILDREN - IS THE COVID-19 PANDEMIC IMPACTING TEESSIDE DIFFERENTLY?}

Matthew Davidson, Shashwat Saran, Qasim Mansoor, Thomas Salisbury. UK

\subsection{6/bmjpo-2021-RCPCH.247}

Background An article published by Sidpra J et al, in ADC in July 2020 reported an increase of approximately 1500\% in the incidence of abusive head trauma (AHT) in children, understandably shocking paediatricians nationally.

Objectives We wanted to see the impact of lockdown on referrals made to our hospital (University Hospital of North Tees, Stockton, UK) for children suspected to have non-accidental injuries (NAI) or AHT.

Methods We audited the records of children referred with suspected NAI during the first lockdown period (23rd of March to 4th of July 2020) and compared it with the preceding year (23rd of March to 4th of July 2019)

As children under two are more likely to suffer AHT, these cases were identified by two auditors and then a single auditor reviewed these case records in detail.

Results Total of thirty-one children in 2019 \& twenty-five in 2020 were referred for Child Protection Medical Assessments. Eleven children - six in 2019 and five in 2020 - were less than two years old. Of these, four were girls and seven boys. Five children were less than three months old

Nine children were referred with suspicious bruising, one was the sibling of an index child with NAI and for one, an allegation was made of rough handling.

Two children with skull fractures and one with a rib fracture (on follow up scan) were identified on radiological investigations. Hematological investigations and ophthalmic findings were unremarkable for all the children.

All children were seen within 24 hours of a referral being made. In all cases, an interim report was provided on the same day and a final report within seven working days. All children were investigated as per RCPCH guidelines.

Seven out of eleven children referred for NAI assessment were concluded to have probable non-accidental injuries three in 2019, and four in 2020.

Our neighbouring hospital received five and four referrals for suspected NAI in children under 2 years old in the same period of time in 2019 and 2020 respectively. One of these children in 2020 had retinal haemorrhages associated with AHT and a skull fracture.

Conclusions Our audit of children referred for NAI medical assessment does not suggest any significant difference between numbers in 2019 and 2020. This is in contrast with what colleagues from UCL, London have reported, and surprising given the high levels of socioeconomic deprivation in our area, which is usually associated with a higher incidence of child abuse.

In order to understand national trends, we recommend other areas of the United Kingdom to review their data to establish whether this pandemic and associated lockdowns are impacting children in Teesside differently.

\section{PROMOTING SAFE SLEEPING IN A NEONATAL UNIT}

Catherine Taylor, Amna Suliman, Martha Jones, Louise Mawby, Esmira Jafarova, Sarah Panjwani. UK

\subsection{6/bmjpo-2021-RCPCH.248}

Background Sudden Infant Death Syndrome (SIDS) has been significantly reduced in the UK following the introduction of the 'back to sleep campaign' and ongoing efforts in raising public awareness of the importance of a safe sleep environment. Babies born prematurely or who have ongoing health conditions are known to be at higher risk. Local data suggests most babies that die from SIDS locally have modifiable risk factors.

On neonatal units, babies may initially require prone positioning and the presence of equipment within an incubator, however they should be transitioned to a safe sleep environment prior to discharge. Research has shown that modelling a safe sleep environment on neonatal units can improve parental compliance and patient safety after discharge. 